\title{
Body image and quality of life among colorectal cancer survivors: A literature review
}

The Nethersole School of Nursing, Faculty of Medicine, The Chinese University of Hong Kong, Hong Kong SAR

\section{Background}

Colorectal cancer is the third most common cancer diagnosed worldwide, and patients' quality of life and body image are significantly worsened along the treatment process. However, there is currently very limited evidence regarding the association between these two outcomes among colorectal cancer survivors, especially those with a stoma.

\section{Objective}

This review aims to identify, summarise and critically appraise current literature investigating body image and quality of life, as well as the association between these two outcomes among colorectal cancer survivors.

\section{Methods}

\section{Search strategy}

A comprehensive search was conducted to retrieve relevant published studies from four electronic databases including MEDLINE, PsycINFO, CINAHL, and British Nursing Index.

\section{Keywords}

Colorectal cancer, colorectal carcinoma, colorectal malignancy, colostomy, stoma, quality of life and body image were used in combination.

\section{Inclusion and exclusion criteria}

This review considered all studies that (1) examined outcomes of either body image or quality of life among colorectal cancer patients, (2) were written in the English language and (3) were published from 2007 to 2017. Studies were excluded if they (1) do not specify the reason participants having a colorectal surgery or stoma were a result of colorectal cancer, or (2) involved colorectal cancer patients who had a colostomy or a stoma, but with data indistinguishable from the whole sample population.

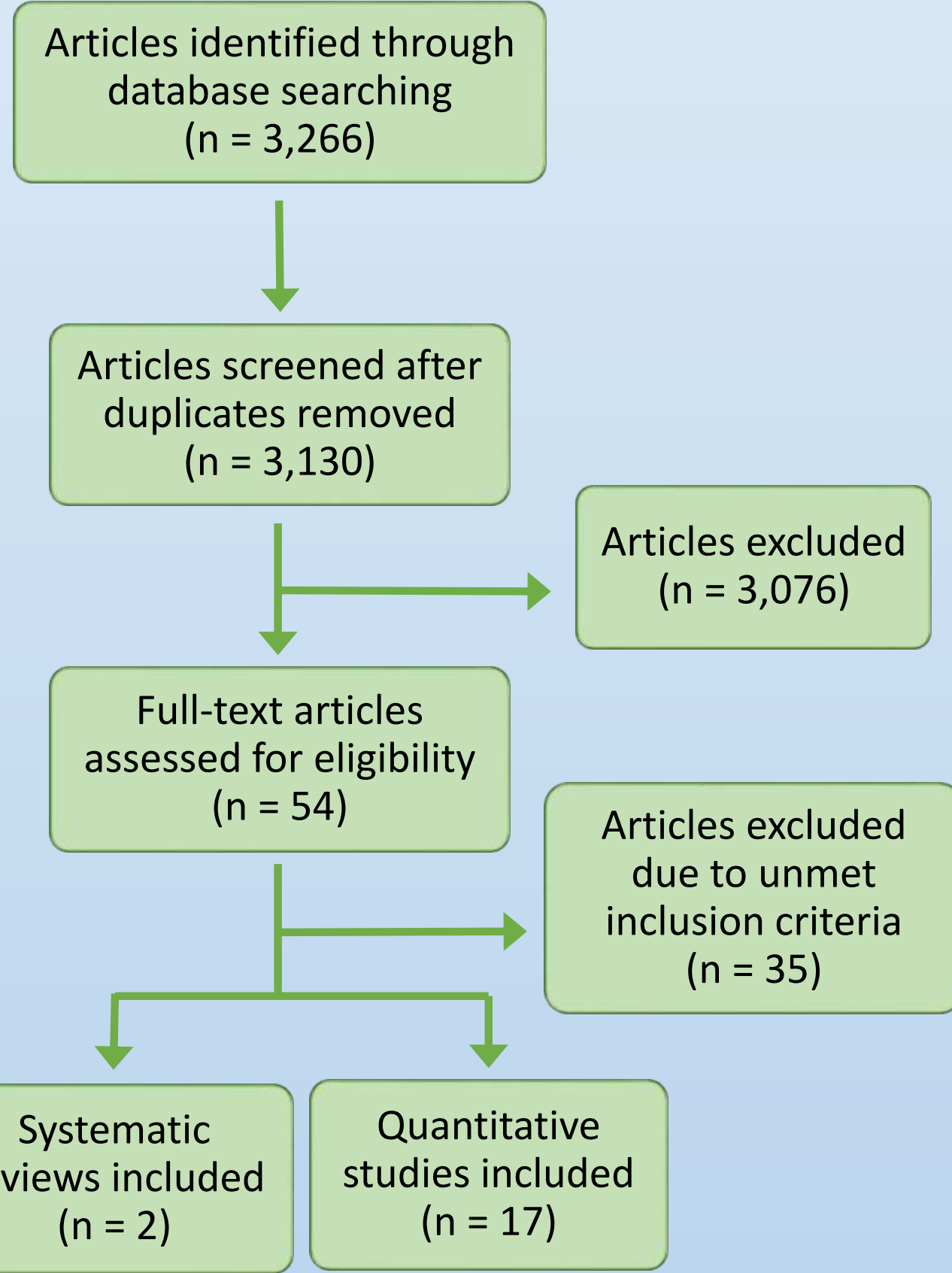

\section{Results}

A total of 3,266 potentially relevant articles were identified from the search, and 19 studies were included in this review. Colorectal cancer survivors generally reported having poorer quality of life than the general population, but improvement was observed with time. However, those survivors with stoma reported to have significantly lower body image which was persistent and worsen over time. Similarly, survivors with stoma had poorer quality of life than those without it, yet some studies reported no significant difference. Also, body image disturbance was found to be negatively correlated with emotional aspect of quality of life, but there was very

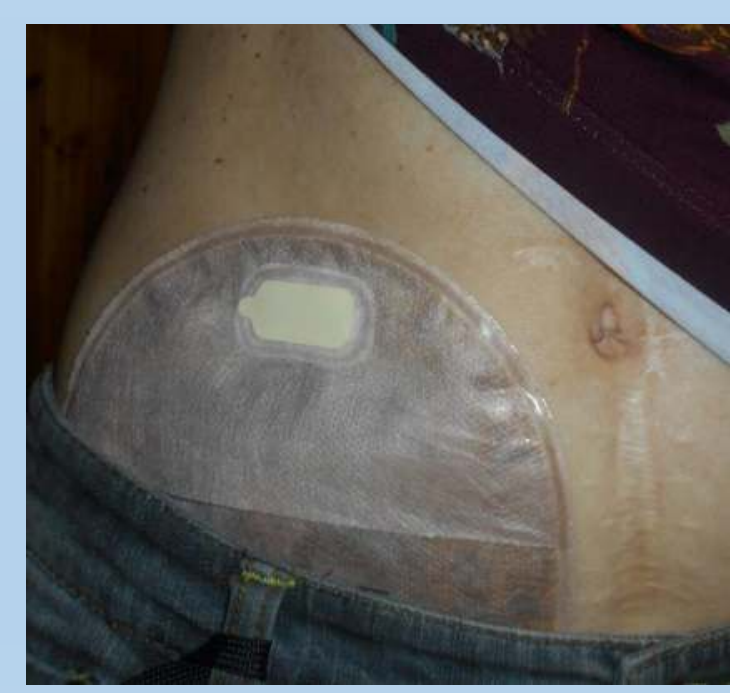
limited evidence supporting this finding.

\section{Conclusion}

Colorectal cancer survivors, especially those with stoma, reported poorer body image and quality of life. Appropriate stoma-specific nursing interventions should be identified to address their needs. Future studies in various cultural context should also be conducted. 